\title{
DETERMINATION OF QUADRATIC EXTENSIONS OF LINEARLY COMPACT FIELDS BY NORM GROUPS
}

\author{
HOYT D. WARNER ${ }^{1}$
}

\begin{abstract}
It is shown that quadratic extensions of a field not of characteristic two, which is linearly compact at a valuation, are determined by their groups of norms, provided the residue field has a unique quadratic extension and is perfect if of characteristic two. It is indicated that Henselian can replace linearly compact in some cases. Necessity of the condition on the residue field is shown.
\end{abstract}

1. In this brief paper we shall apply the techniques and results of the paper Quadratic extensions of linearly compact fields by Ron Brown and myself (referred to below as [BW]) to prove the following result:

THEOREM 1. Let $F$ be a field of characteristic $\operatorname{char}(F) \neq 2$. Let $v$ be a (nonarchimedean) valuation on $F$ with arbitrary value group $\Gamma_{F}$ and residue field $k_{F}$; assume only that $k_{F}$ is perfect if $\operatorname{char}\left(k_{F}\right)=2$.

Suppose that $F$ is linearly compact at $v$ and that $k_{F}$ has a unique quadratic extension. Then for $K_{1}$ and $K_{2}$ quadratic extensions of $F$,

$$
K_{1} \simeq K_{2} \text { if and only if } N_{1} K_{1}=N_{2} K_{2} .
$$

(Here $N_{i}$ denotes the norm map $K_{i} \rightarrow F$.) For definition and properties of linear compactness, see [BW] or [Bour].

All the hypotheses of Theorem 1 are satisfied by any classical local field of characteristic not two. Indeed Theorem 1 is a generalization of a special case of the local class field theorem which says that an abelian extension of a local field is determined by its group of norms. The conclusion of Theorem 1 is equivalent to the assertion that a binary quadratic form over $F$ is determined up to equivalence by the elements of $F$ which it represents. A straightforward application of the Global Squares Theorem extends this result to the global case, obtaining the well-known result that over any local or global field of characteristic not two, binary quadratic forms are equivalent if and only if they represent the same elements.

Received by the editors July 9, 1971.

AMS 1970 subject classifications. Primary 12B10, 12J10, 12B25; Secondary 10C05, $12 \mathrm{~A} 25,12 \mathrm{~J} 20$.

Key words and phrases. Quadratic extensions, linearly compact fields, norm groups, group valuation.

${ }^{1}$ Research done while the author was a Postdoctoral Fellow at the University of Illinois.

(c) American Mathematical Society 1972 
The hypothesis of linear compactness is needed to handle the wildly ramified case, i.e. ramified quadratic extensions when $\operatorname{char}\left(k_{F}\right)=2$. If $\operatorname{char}\left(k_{F}\right) \neq 2$ then $F$ Henselian at $v$ suffices-see Remark 2.3 below.

We summarize in $\$ 2$ the concepts and results needed from [BW]; the proof of Theorem 1 is in $\S 3$. $\S 4$ contains examples to show the necessity of the "unique quadratic extension of $k_{F}$ " hypothesis in Theorem 1.

2. Notation, summary of results from [BW]. Throughout $\S \S 2$ and 3 , $F$ will denote a field of characteristic not two, and $v$ will denote a (nonarchimedean) valuation on $F$ with value group $\Gamma_{F}$, a linearly ordered abelian group written additively, and residue field $k_{F}$; we assume $k_{F}$ is perfect if $\operatorname{char}\left(k_{F}\right)=2$. For any field $L, L^{\times}$will denote its multiplicative group of nonzero elements. $Z$ denotes the rational integers.

Define a linearly ordered set $\Lambda_{F}$ by

$$
\Lambda_{F}=\left\{0^{*}\right\} \cup\left\{\alpha \in \Gamma_{F} \mid \alpha \geqq 0\right\}
$$

where $0^{*}$ is a formal symbol, $\Lambda_{F} \cap \Gamma_{F}$ inherits its order from $\Gamma_{F}$, and $0 * \leqq \alpha$ for all $\alpha \in \Lambda_{F}$.

Convention. In all algebraic formulas appearing below we regard $\Lambda_{F}$ as embedded in $\Gamma_{F}$ by $0 * \rightarrow 0, \alpha \rightarrow \alpha$ for $\alpha \geqq 0$.

The map $v^{\times}: F^{\times} \rightarrow \Lambda_{F} \cup\{\infty\}$ defined by

$$
\begin{aligned}
v^{\times}(a) & =0^{*} & & \text { if } v(a) \neq 0, \\
& =v(1-a) & & \text { if } v(a)=0,
\end{aligned}
$$

is a group valuation on the multiplicative group $F^{\times}$. That is, $v^{\times}$satisfies:

(a) $v^{\times}(a)=\infty$ if and only if $a=1$.

(b) $v^{\times}(a b) \geqq \min \left\{v^{\times}(a), v^{\times}(b)\right\}$.

(c) $v^{\times}\left(a^{-1}\right)=v^{\times}(a)$.

Theorems (1.1) and (1.3) of [BW] imply that $v^{\times}$naturally induces group valuations on the square factor group $F^{\times} / F^{\times 2}$, and the norm factor group $F^{\times} / N K^{\times}$of a quadratic extension $K$ of $F$, provided $F$ is linearly compact at $v$ (or, in certain special cases, if $F$ is Henselian at $v$-see Remark 2.3). Those theorems do more, giving explicit calculations of these groups as valued groups. In the theorem below we extract in convenient form the information needed for the proof of Theorem 1 .

THEOREM 2. Let $F$ and $v$ satisfy the hypotheses stated above. Suppose, further, that $F$ is linearly compact at $v$. Then:

A. The group valuation $v^{\times}$on $F^{\times}$induces a group valuation $v^{\mathrm{sq}}: F^{\times} / F^{\times 2} \rightarrow$ $\Lambda_{F} \cup\{\infty\}$ by

$$
v^{\mathrm{sq}}\left(a F^{\times 2}\right)=\max \left\{v^{\times}\left(a \cdot c^{2}\right) \mid c \in F^{\times}\right\} .
$$


The only values which can be taken on by $v^{\mathrm{sq}}$ are:

$$
\begin{array}{rlrl}
v^{\mathrm{sq}}\left(a F^{\times 2}\right) & =\infty & & \text { if and only if } a \in F^{\times 2} ; \\
& =0^{*} & & \text { if and only if } v(a) \notin 2 \Gamma_{F} ; \\
& =v(4) & & \text { if and only if } F \sqrt{ } a / F \text { is } \\
& \text { unramified; } \\
& =\alpha, \text { for } 0<\alpha<v(4), \alpha \notin 2 \Gamma_{F} & & \begin{array}{c}
\text { only if } v(4)>0, \text { i.e. } \\
\operatorname{char}\left(k_{F}\right)=2 .
\end{array}
\end{array}
$$

Also, the set $\left\{a F^{\times 2} \mid v^{\mathrm{sq}}\left(a F^{\times 2}\right)=v(4)\right\}$ is in one-one correspondence with the set of quadratic extensions of $k_{F}$.

B. Let $K=F \sqrt{ } d, d$ a nonsquare in $F$. Let $w$ be the unique extension to $K$ of the valuation $v$, with value group $\Gamma_{K}$ and residue field $k_{K}$. The group valuation $v^{\times}$on $F^{\times}$induces a group valuation $v^{N}$ on $F^{\times} / N K^{\times}$(where $N$ denotes $\left.N_{K / F}\right)$ by

$$
v^{N}\left(a N K^{\times}\right)=\max \left\{v^{\times}(a \cdot N t) \mid t \in K^{\times}\right\} .
$$

The only values which can be taken on by $v^{N}$ are:

$$
\begin{array}{rlrl}
v^{N}\left(a N K^{\times}\right) & =\infty & & \text { if and only if } a \in N K^{\times} ; \\
& =0^{*} & & \text { if and only if } v(a) \notin 2 \Gamma_{K} ; \\
& =v(4)-v^{\mathrm{sq}}\left(d F^{\times 2}\right) & & \text { this }=0 \text { by our convention when } \\
& & \operatorname{char}\left(k_{F}\right) \neq 2 ; \\
& =\alpha, \text { for } 0<\alpha<v(4)-v^{\mathrm{sq}}\left(d F^{\times 2}\right) \text { and } a \notin 2 \Gamma_{K} .
\end{array}
$$

Also, when $K$ is ramified over $F$, the set $\left\{a N K^{\times} \mid v^{N}\left(a N K^{\times}\right)=v(4)-v^{\mathrm{sq}}\left(d F^{\times 2}\right)\right\}$ is in one-one correspondence with the quadratic extensions of $k_{F}$.

C. With $K$ as in $\mathrm{B}$, one has

(1) $2 \Gamma_{K}=2 \Gamma_{F}+Z \cdot v(d)+Z \cdot v^{\mathrm{sq}}\left(d F^{\times 2}\right)$;

(2) if $0<v^{\times}(a)<v(4)-v^{\mathrm{sq}}\left(d F^{\times 2}\right)$ and $v^{\times}(a) \notin 2 \Gamma_{K}$, then $a \notin N K^{\times}$.

Proof. A and B follow from Theorem (1.1) and (1.3) of [BW], respectively (see also $\S 5$ ); note that if $\operatorname{char}\left(k_{F}\right)=2$, the nonzero elements of the group $k_{F}^{+} /\left\{a^{2}-a \mid a \in k_{F}\right\}$ are in one-one correspondence with the quadratic extensions of $k_{F} . \mathrm{C}(1)$ is (2) of [BW]; C(2) is part of Lemma (3.2) of $[\mathrm{BW}]$.

REMARK 2.2. Assume the situation of Theorem 2. Since for $a \in F^{\times}$, $v^{\times}(a) \leqq v^{\mathrm{sq}}\left(a F^{\times 2}\right) \leqq v^{\mathrm{v}}\left(a \cdot N K^{\times}\right)$where $K=F \sqrt{ } d$ is a quadratic extension of $F$, one sees immediately that $v^{\times}(a)>v(4)$ implies $a \in F^{\times 2}, v^{\times}(a)>v(4)-$ $v^{\mathrm{sq}}\left(d F^{\times 2}\right)$ implies $a \in N K^{\times}$. Moreover, if $\operatorname{char}\left(k_{F}\right)=2$ and $K=F \sqrt{ } d$ is unramified over $F$ so that $v^{\text {sq }}\left(d F^{\times 2}\right)=v(4)$, then the only values which $v^{N}$ can take on are $0^{*}$ and $\infty\left(\right.$ as $v^{\mathrm{sa}}\left(a F^{\times 2}\right)=0$ is impossible as $k_{F}$ is perfect, so also $v^{N}\left(a N K^{\times}\right)=0$ is impossible). This means that $a \in N K^{\times}$if and only if $v(a) \in 2 \Gamma_{K}=2 \Gamma_{F}$ (since $K$ is unramified over $\left.F\right)$. 
REMARK 2.3. As shown in $\S 5$ of [BW], the conclusions of Theorem 2 still hold when $F$ is only Henselian at $v$, provided either $\operatorname{char}\left(k_{F}\right) \neq 2$ or the isolated subgroup of $\Gamma_{F}$ which is generated by $v(2)$ is discrete. Therefore for such fields the conclusion of Theorem 1 also holds.

3. Proof of Theorem 1. We continue the hypotheses on $F$ from $\S 2$. We further assume that $F$ is linearly compact at $v$, or at least that the conclusions of Theorem 2 are valid (see Remark 2.3 for cases when this holds without the hypothesis that $F$ is linearly compact at $v$ ).

To prove Theorem 1, it suffices to show the following: if, in addition to the above hypotheses, $k_{F}$ possesses a unique quadratic extension, then for nonsquares $d_{1}, d_{2} \in F, d_{1} F^{\times 2} \neq d_{2} F^{\times 2}$ implies $N_{1}\left(F \sqrt{ } d_{1} \times\right) \neq N_{2}\left(F \sqrt{ } d_{2}^{\times}\right.$) (for $N_{i}$ the norm map $F \sqrt{ } d_{i} \rightarrow F$ ).

Notation. With $d_{1}, d_{2}$ as above, let $K_{i}=F \sqrt{ } d_{i}, i=1,2$. Let $\Gamma_{i}, k_{i}$ denote the value group and residue field, respectively, of the unique extension $w_{i}$ of $v$ to a valuation on $K_{i}$, for $i=1,2$.

Normalizations. We may assume without loss that $v^{\mathrm{sq}}\left(d_{1} F^{\times 2}\right) \leqq$ $v^{\text {sq }}\left(d_{2} F^{\times 2}\right)$. Moreover, replacing $d_{2}$ by $d_{2} c^{2}$ if necessary, we may assume that $v^{\times}\left(d_{2}\right)=v^{\mathrm{sq}}\left(d_{2} F^{\times 2}\right)$ and that $v\left(d_{2}\right)>0$ if $v^{\mathrm{sq}}\left(d_{2} F^{\times 2}\right)=0 *$. These normalizations (plus our convention on $0^{*}$, see $\S 2$ ) imply that $v^{\mathrm{sq}}\left(d_{2} F^{\times 2}\right)+2 \Gamma_{F}=$ $v\left(1-d_{2}\right)+2 \Gamma_{F}$ in all cases. Finally, replacing $d_{1}$ by $d_{1} c^{2}$ if necessary, where $v^{\times}(c) \geqq v^{\mathrm{sq}}\left(d_{1} d_{2} F^{\times 2}\right) \geqq v^{\mathrm{sq}}\left(d_{1} F^{\times 2}\right)$ (which does not change $\left.v^{\mathrm{sq}}\left(d_{1} F^{\times 2}\right)\right)$, we may also assume $v^{\times}\left(d_{1} d_{2}\right)=v^{\mathrm{sq}}\left(d_{1} d_{2} F^{\times 2}\right)$.

To begin the proof, recall from valuation theory, cf. [Sch], that from the definition of the unique extension to $K_{i}$ of the valuation $v$ on $F$ it follows that $v\left(N K_{i} \times\right)=2 \Gamma_{i}$. Therefore if $2 \Gamma_{1} \neq 2 \Gamma_{2}$, surely $N K_{1} \times \neq N K_{2} \times$. Therefore we henceforth assume $2 \Gamma_{1}=2 \Gamma_{2}$.

Next, for essentially obvious reasons, it cannot be true that both $K_{1}$ and $K_{2}$ are unramified over $F$. For, as $k_{F}$ has a unique quadratic extension, $F$ has a unique unramified extension [explicitly, by Theorem $2 \mathrm{~A}, K_{i}$ unramified means $v^{\mathrm{sq}}\left(d_{i} F^{\times 2}\right)=v(4)$ for $i=1,2$, but by the last part of Theorem $2 \mathrm{~A}$ and the existence of only one quadratic extension of $k_{F}$, this implies $d_{1} F^{\times 2}=d_{2} F^{\times 2}$, a contradiction]. Hence we will further assume, for the remainder of the proof, that $K_{1}$ and $K_{2}$ are ramified over $F$.

We distinguish two main cases.

Case I. $\quad v^{\mathrm{sq}}\left(d_{1} d_{2} F^{\times 2}\right) \notin 2 \Gamma_{1}=2 \Gamma_{2}$.

Case II. $v^{\mathrm{sq}}\left(d_{1} d_{2} F^{\times 2}\right) \in 2 \Gamma_{1}=2 \Gamma_{2}$.

In each case we will show the existence of an element $u \in F$ such that $u \in N K_{1} \times \cdot N K_{2} \times$ but $u \notin N K_{2} \times$, proving $N K_{1} \times \neq N K_{2} \times$.

Proof of CASE I. Let

$$
u=\frac{1-\left(d_{2}\right)^{2} d_{1}}{\left(-d_{1}\right)\left(-d_{2}\right)\left(1-d_{2}\right)}=1+\frac{1-d_{1} d_{2}}{d_{1} d_{2}\left(1-d_{2}\right)} .
$$


By construction, $u \in N K_{1} \times \cdot N K_{2} \times$, since $\left(1-\left(d_{2}\right)^{2} d_{1}\right) /\left(-d_{1}\right) \in N K_{1} \times$ and $\left(-d_{2}\right)\left(1-d_{2}\right) \in N K_{2} \times$. Since $\quad v^{\mathrm{sq}}\left(d_{1} F^{\times 2}\right) \leqq v^{\mathrm{sq}}\left(d_{1} d_{2} F^{\times 2}\right) \notin 2 \Gamma_{1} \quad$ while $v^{\mathrm{sq}}\left(d_{1} F^{\times 2}\right) \in 2 \Gamma_{1}$, we must have $0^{*} \leqq v^{\mathrm{sq}}\left(d_{1} F^{\times 2}\right)<v^{\mathrm{sq}}\left(d_{1} d_{2} F^{\times 2}\right)=v^{\times}\left(d_{1} d_{2}\right)$ so that $v^{\times}\left(d_{1} d_{2}\right) \geqq 0$ and $v\left(d_{1} d_{2}\right)=0$. Also, this implies $v^{\mathrm{sq}}\left(d_{2} F^{\times 2}\right)=v^{\mathrm{sq}}\left(d_{1} F^{\times 2}\right)$ (since $\left.v^{\mathrm{sq}}\left(d_{1} F^{\times 2} \cdot d_{2} F^{\times 2}\right)>v^{\mathrm{sq}}\left(d_{1} F^{\times 2}\right)=\min \left\{v^{\mathrm{sq}}\left(d_{1} F^{\times 2}\right), v^{\mathrm{sq}}\left(d_{2} F^{\times 2}\right)\right\}\right)$ and hence $v\left(\left(1-d_{1} d_{2}\right) / d_{1} d_{2}\left(1-d_{2}\right)\right)=v^{\times}\left(d_{1} d_{2}\right)-v^{\times}\left(d_{2}\right)=v^{\mathrm{sq}}\left(d_{1} d_{2} F^{\times 2}\right)-v^{\mathrm{sq}}\left(d_{2} F^{\times 2}\right)>0$ so that $v^{\times}(u)=v^{\mathrm{sq}}\left(d_{1} d_{2} F^{\times 2}\right)-v^{\mathrm{sq}}\left(d_{2} F^{\times 2}\right) \notin 2 \Gamma_{2} \quad$ (since $v^{\mathrm{sq}}\left(d_{2} F^{\times 2}\right) \in 2 \Gamma_{2}$ (by Theorem $2 \mathrm{C}(1))$, while $\left.v^{\mathrm{sq}}\left(d_{1} d_{2} F^{\times 2}\right) \notin 2 \Gamma_{2}\right)$. But further, $v^{\mathrm{sq}}\left(d_{1} d_{2} F^{\times 2}\right) \neq$ $v(4)$ (as $\left.v(4) \in 2 \Gamma_{2}\right)$ so that we have $0<v^{\times}(u)<v(4)$ and $v^{\times}(u) \notin 2 \Gamma_{2}$. Hence by Theorem $2 \mathrm{C}(2), u \notin N K_{2} \times$.

Proof of CASE II. We know from Theorem 2B that since $k_{F}$ has a unique quadratic extension and $K_{2}$ is ramified over $F$, there is a (unique) coset $u \cdot N K_{2} \times$ with $v^{N}\left(u \cdot N K_{2} \times\right)=v(4)-v^{\mathrm{sq}}\left(d_{2} F^{\times 2}\right) \neq \infty$. By replacing $u$ by $u \cdot N(t)$ if necessary we may assume $v^{\times}(u)=v(4)-v^{\mathrm{sq}}\left(d_{2} F^{\times 2}\right)$. We shall show that $u \in N K_{1} \times \cdot N K_{2} \times$ which, together with the fact that $u \notin N K_{2} \times$, will complete the proof.

To show $u \in N K_{1} \times \cdot N K_{2} \times$ we return to quadratic forms and observe that $u \in N K_{1} \times \cdot N K_{2} \times$ is equivalent to the assertion that the form

$$
\psi(x, y, w, z)=x^{2}-d_{2} y^{2}-u\left(w^{2}-d_{1} z^{2}\right)
$$

is isotropic, i.e. represents zero. For, $x^{2}-d_{2} y^{2}$ and $w^{2}-d_{1} z^{2}$ are anisotropic (as $\left.d_{i} \notin F^{\times 2}, v=1,2\right)$. So $0=\psi(a, b, e, f)$ and not all of $a, b, e, f=0$ implies $(e, f) \neq(0,0), e^{2}-d_{1} f^{2} \neq 0$, and $u=\left(a^{2}-d_{2} b^{2}\right) /\left(e^{2}-d_{1} f^{2}\right) \in N K_{1} \times \cdot N K_{2} \times$.

To prove $\psi$ isotropic, we transform it to an equivalent form by the following linear change of variables

$$
\begin{gathered}
x_{1}=x-d_{2} y+u d_{1} d_{2} z, \quad y_{1}=x-y+u d_{1} z, \\
w_{1}=\left(1-d_{2}\right) y+d_{1} d_{2} z, \quad z_{1}=w .
\end{gathered}
$$

The determinant of this transformation is

$$
\left(1-d_{2}\left(u d_{1} d_{2}\right)\right)\left(u-\left(-d_{2}\right)\left(1-d_{2}\right)\right) \neq 0,
$$

since $\left(-d_{2}\right)\left(1-d_{2}\right) \in N K_{2}$ but $u \notin N K_{2}$ (and $d_{2} \notin F^{\times 2}$ ). With this change of variables,

for

$$
\psi\left(x_{1}, y_{1}, w_{1}, z_{1}\right)=\psi^{\prime}(x, y, z, w)
$$

$$
\psi^{\prime}(x, y, w, z)=\left(1-d_{2}\right)\left(x^{2}-d_{2}{ }^{*} y^{2}\right)+u d_{1}\left(w^{2}-d_{1} d_{2} d_{2}{ }^{*} z^{2}\right)
$$

where $d_{2}{ }^{*}=u\left(1-d_{2}\right)+d_{2}$, so that $1-d_{2}{ }^{*}=\left(1-d_{2}\right)(1-u)$.

Claim. $v^{\mathrm{sq}}\left(d_{2}{ }^{*} F^{\times 2}\right)=v(4)$.

SubProof. First, $v\left(d_{2}{ }^{*}\right)=0$ : If $\operatorname{char}\left(k_{F}\right)=2$ then $v(1-u)=v^{\times}(u)=$ $v(4)-v^{\mathrm{sq}}\left(d_{2} F^{\times 2}\right)>0$ (as $K_{2}$ is not unramified over $F$, i.e. $v^{\mathrm{sq}}\left(d_{2} F^{\times 2}\right) \neq v(4)$ ). Hence, as $v\left(d_{2}\right) \geqq 0$ by our normalization, $v\left((1-u) d_{2}\right)>0$ so $v\left(d_{2}{ }^{*}\right)=$ $v\left((1-u) d_{2}+u\right)=v(u)=0$. If $\operatorname{char}\left(k_{F}\right) \neq 2, v\left(d_{2} \times\right) \geqq 0$ in any case as 
$v\left(1-d_{2}\right) \geqq 0, v\left(d_{2}\right) \geqq 0$ by normalization. If then $v\left(d_{2}{ }^{*}\right)=v\left(u\left(1-d_{2}\right)+d_{2}\right)>$ $0=v(4)$, it would follow that $v\left(1-u^{-1}\left(-d_{2}\right)\left(1-d_{2}\right)^{-1}\right)>0=v(4)$ (using $\left.v(u)=0, v\left(1-d_{2}\right)=0\right)$ which implies $v^{\times}\left(u^{-1}\left(-d_{2}\right)\left(1-d_{2}\right)^{-1}\right)>v(4)$. But then by Theorem $2 \mathrm{~A}, u^{-1}\left(-d_{2}\right)\left(1-d_{2}\right)^{-1}=c^{2} \in F^{\times 2}$, so $u=\left(c^{-1}\right)^{2}\left(-d_{2}\right)\left(1-d_{2}\right)^{-1} \in$ $N K_{2} \times$, a contradiction.

Therefore, $v^{\times}\left(d_{2}{ }^{*}\right)=v\left(1-d_{2}{ }^{*}\right)=v\left(1-d_{2}\right)+v(1-u)=v^{\times}\left(d_{2}\right)+v^{\times}(u)=v(4)$ by normalization of $d_{2}$ and choice of $u$. If $v^{\mathrm{sq}}\left(d_{2}{ }^{*}\right)>v(4)$, then $d_{2}{ }^{*}=c^{2} \in$ $F^{\times 2}$, but then $c^{2}=d_{2}{ }^{*}=u\left(1-d_{2}\right)+d_{2}$ implies $u=\left(c^{2}-d_{2}\right)\left(1-d_{2}\right)^{-1} \in N K_{2}{ }^{\times}$, a contradiction. The claim is proven.

With the claim, we are now done in case $v^{\mathrm{sq}}\left(d_{1} d_{2} F^{\times 2}\right)=v(4)$, which will necessarily occur when $\operatorname{char}\left(k_{F}\right) \neq 2$ because $2 \Gamma_{1}=2 \Gamma_{2}$ and $K_{1}, K_{2}$ are ramified over $F$. For, $v^{\text {sq }}\left(d_{1} d_{2} F^{\times 2}\right)=v(4)$ implies (Theorem $2 \mathrm{~A}$ ) that each of the cosets $d_{1} d_{2} F^{\times 2}$ and $d_{2}{ }^{*} F^{\times 2}$ must correspond to the unique quadratic extension of $k_{F}$. Thus the cosets are equal, so that $d_{1} d_{2} d_{2}{ }^{*} \in F^{\times 2}, w^{2}-$ $d_{1} d_{2} d_{2}{ }^{*} z^{2}$ is isotropic, and $\psi^{\prime}$, hence $\psi$, is isotropic.

We must finally consider the case when $v^{\text {sq }}\left(d_{1} d_{2} F^{\times 2}\right)<v(4)$, in which case $v^{\mathrm{sq}}\left(d_{1} d_{2} d_{2}{ }^{*} F^{\times 2}\right)=v^{\mathrm{sq}}\left(d_{1} d_{2} F^{\times 2}\right)$ which is in $2 \Gamma_{1}=2 \Gamma_{2}$ by our standing Case II hypothesis. Moreover, we are in the wildly ramified case, with $\operatorname{char}\left(k_{F}\right)=2$ (see previous paragraph). Now, by Remark $2.2, v^{\mathrm{sq}}\left(d_{2}{ }^{*} F^{\times 2}\right)=v(4)$ implies that the form $x^{2}-d_{2}{ }^{*} y^{2}$, which is the norm form of $F \sqrt{ } d_{2}{ }^{*}$, represents all $a \in F$ with $v(a) \in 2 \Gamma_{F}$, and hence the first subform $\left(1-d_{2}\right)\left(x^{2}-d_{2}{ }^{*} y^{2}\right)$ of $\psi^{\prime}$ represents all $a \in F$ with $v(a) \in v\left(1-d_{2}\right)+2 \Gamma_{F}=v^{\mathrm{sq}}\left(d_{2}\right)+2 \Gamma_{F}$ (by our normalization). Therefore to conclude the proof it suffices to show that the second subform, $u d_{1}\left(w^{2}-d_{1} d_{2} d_{2}{ }^{*} z^{2}\right)$, also represents some element of value in $v^{\mathrm{sq}}\left(d_{2} F^{\times 2}\right)$. The values of elements represented by $u d_{1}\left(w^{2}-d_{1} d_{2} d_{2}{ }^{*} z^{2}\right)$ are, by Theorem $2 \mathrm{C}(1)$, all of the set $v\left(d_{1}\right)+2 \Gamma_{F}+Z \cdot v\left(d_{1} d_{2} d_{2}{ }^{*}\right)+$ $Z \cdot v^{\mathrm{sq}}\left(d_{1} d_{2} d_{2}{ }^{*} F^{\times 2}\right)=v\left(d_{1}\right)+2 \Gamma_{F}+Z \cdot v\left(d_{1} d_{2}\right)+Z v^{\mathrm{sq}}\left(d_{1} d_{2} F^{\times 2}\right) \quad\left(\right.$ as $v\left(d_{2}{ }^{*}\right)=0$, and by the beginning of this paragraph). Thus we must show that:

$\left(v^{\mathrm{sq}}\left(d_{2} F^{\times 2}\right)+2 \Gamma_{F}\right) \cap\left(v\left(d_{1}\right)+2 \Gamma_{F}+Z \cdot v\left(d_{1} d_{2}\right)+Z \cdot v^{\mathrm{sq}}\left(d_{1} d_{2} F^{\times 2}\right)\right) \neq \varnothing$ or (as we have cosets $\bmod 2 \Gamma_{F}$ )

$$
v^{\mathrm{sq}}\left(d_{2} F^{\times 2}\right)+v\left(d_{1}\right) \in 2 \Gamma_{F}+Z \cdot v\left(d_{1} d_{2}\right)+Z \cdot v^{\mathrm{sq}}\left(d_{1} d_{2} F^{\times 2}\right) .
$$

Since $v^{\mathrm{sq}}\left(d_{2} F^{\times 2}\right)+v\left(d_{1}\right) \in 2 \Gamma_{1}+2 \Gamma_{2}=2 \Gamma_{2}+2 \Gamma_{2}=2 \Gamma_{2}$, it suffices to show that the right side of (2) equals $2 \Gamma_{2}$. First, if $v^{\mathrm{sq}}\left(d_{1} d_{2} F^{\times 2}\right)>0^{*}$, then $0<$ $v^{\mathrm{sq}}\left(d_{1} d_{2} F^{\times 2}\right)<v(4)$ (it is $<v(4)$ by our assumption, $>0$ since $=0$ is impossible when $\left.\operatorname{char}\left(k_{F}\right)=2\right)$. Hence by Theorem $2 \mathrm{~A}, v^{\mathrm{sq}}\left(d_{1} d_{2} F^{\times 2}\right) \notin 2 \Gamma_{F}$, and $v\left(d_{1} d_{2}\right)=0$. But $v^{\text {sq }}\left(d_{1} d_{2} F^{\times 2}\right) \in 2 \Gamma_{2}$ by our standing Case II hypothesis, so the right side of (2) is equal to $2 \Gamma_{2}$. Finally, if $v^{\text {sq }}\left(d_{1} d_{w} F^{2}\right)=0^{*}$, then since $v^{\mathrm{sq}}\left(d_{1} F^{\times 2}\right) \leqq v^{\mathrm{sq}}\left(d_{2} F^{\times 2}\right)$, we must have $0^{*}=v^{\mathrm{sq}}\left(d_{1} F^{\times 2}\right)<0<v^{\mathrm{sq}}\left(d_{2} F^{\times 2}\right)$ (for $v^{\mathrm{sq}}\left(d_{2} F^{\times 2}\right)=0 *$ would imply, since $2 \Gamma_{1}=2 \Gamma_{2}$, that $v\left(d_{1}\right)+2 \Gamma_{F}=$ $v\left(d_{2}\right)+2 \Gamma_{F}$, so $v\left(d_{1} d_{2}\right) \in 2 \Gamma_{F}$ and $v^{\mathrm{sq}}\left(d_{1} d_{2} F^{\times 2}\right)>0 *$, a contradiction). 
Hence, $v\left(d_{1} d_{2}\right)=v\left(d_{1}\right)$, and $2 \Gamma_{2}=2 \Gamma_{1}=2 \Gamma_{F}+Z \cdot v\left(d_{1}\right)=2 \Gamma_{F}+Z \cdot v\left(d_{1} d_{2}\right)$. The theorem is proven.

4. Examples. To show the necessity of the hypothesis in Theorem 1 that $k_{F}$ have exactly one quadratic extension we note the following examples:

EXAMPLE 1. Necessity of "at least one quadratic extension of $k$." Let $k$ denote the quadratic closure of the field $Z_{2}$. That is, $k$ is the smallest algebraic extension of $Z_{2}$ which has no proper extensions of even degree. By a sequence of unramified extensions we can extend the 2-adic numbers $Q_{2}$ to an (infinite) algebraic extension $F_{0}$ such that, if $w_{0}$ denotes the unique extension to $F_{0}$ of the 2-adic valuation $v$ on $Q_{2}, k_{w_{0}}=k$. Let $F$ denote the topological completion of $F_{0}$ with respect to the (discrete) valuation $w_{0}$. Then $F$ is linearly compact at $w$, the unique extension of $w_{0}$ to $F$, and, by [BW, Theorem 1], $F^{\times} / F^{\times 2}=Z / 2 Z \times k_{F}^{+}$, an infinite group since $k_{F}=k$ is infinite. But now, for $K$ any one of the infinitely many quadratic extensions of $F, N K^{\times}=F^{\times}$. For $K$ must be ramified over $F$ (since $k_{F}$ has no quadratic extensions) and $2 \Gamma_{K}=\Gamma_{F}$ for every extension (as $\Gamma_{F}=Z$ ), so the only possible value $\neq \infty$ which could be taken on by $v^{N}$ would be $v(4)-$ $v^{\text {sq }}\left(d \cdot F^{\times 2}\right.$ ) (for $\left.K=F \sqrt{ } d\right)$. However, as stated in Theorem $2.1 \mathrm{~B}$, norm cosets of this value correspond to quadratic extensions of $k_{F}$, which do not exist.

EXAMPLE 2. Necessity of "at most one quadratic extension of $k$ ". Let $F$ be the field of Example 1, let $L$ be the formal power series field $F((x))$ with valuation $v$, then $L$ is linearly compact for $v$, and if $M$ is any one of the infinitely many unramified extensions of $L, N M^{\times}=$ $\{a \in L \mid v(a) \in 2 Z\}$. For, the norm map $N_{M / L}$ induces the norm map from $k_{M}$ to $k_{L}=F$, which is onto as proven in Example 1 above. This, together with the calculation of Theorem 2 of [BW], implies that the only value $\neq \infty$ which $v^{N}\left(a \cdot N M^{\times}\right)$can have is $0^{*}$, which occurs if and only if $v(a) \notin 2 \Gamma_{L}$.

\section{BIBLIOGRAPHY}

[Bour] N. Bourbaki, Eléments de mathématique. Fasc. XXX. Algèbre commutative. Chap. 6: Valuations, Actualités Sci. Indust., no. 1308, Hermann, Paris, 1964. MR 33 \#2660.

[BW] Ron Brown and Hoyt D. Warner, Quadratic extensions of linearly compact fields, Trans. Amer. Math. Soc. 163 (1972), 379-399.

[Sch] O. F. G. Schilling, The theory of valuations, Math. Surveys, no. 4, Amer. Math. Soc., Providence, R.I., 1950. MR 13, 315.

Department of Mathematics, University of Illinois, Urbana, Illinois 61801

Department of Mathematics, Vanderbilt University, Nashville, Tennes See 37203 (Current address) 